\title{
Clinical Role of Lung Ultrasound for the Diagnosis and Prognosis of Coronavirus Disease Pneumonia in Elderly Patients: A Pivotal Study
}

\author{
Guerino Recinella $^{a}$ Giovanni Marasco $^{b}$ Manuel Tufoni ${ }^{b} \quad$ Mara Brizi $^{a}$ \\ Eleonora Evangelisti ${ }^{a} \quad$ Lorenzo Maestri $^{a}$ Marco Fusconi ${ }^{b}$ Pietro Calogero ${ }^{c}$ \\ Donatella Magalottia Marco Zolia \\ aUnit of Internal Medicine, Department of Medical and Surgical Sciences, S. Orsola-Malpighi University

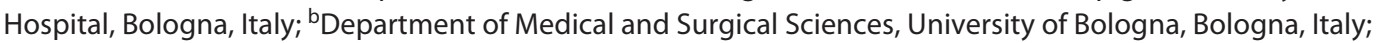 \\ ${ }^{c}$ Acute Geriatric Unit, S. Orsola-Malpighi University Hospital, Bologna, Italy
}

\section{Keywords}

Coronavirus disease $\cdot$ Lung ultrasound $\cdot$ Elderly $\cdot$ Severe acute respiratory syndrome-coronavirus- $2 \cdot$ Aging

\begin{abstract}
Background: Lung ultrasound (LUS) showed a promising role in the diagnosis and monitoring of patients hospitalized for novel coronavirus disease (COVID-19). However, no data are available on its role in elderly patients. Aims: The aim of this study was to evaluate the diagnostic and prognostic role of LUS in elderly patients hospitalized for severe acute respiratory syndrome-coronavirus-2 (SARS-CoV-2) pneumonia. Methods: Consecutive elderly patients (age $>65$ years) hospitalized for COVID-19 were enrolled. Demographics, laboratory, comorbidity, and the clinical features of the patients were collected. All patients underwent LUS on admission to the ward. LUS characteristics have been analyzed. Uni- and multivariate analyses to evaluate predictors for in-hospital death were performed. Results: Thirty-seven hospitalized elderly patients ( 19 men) with a diagnosis of SARS-CoV- 2 infection were consecutively enrolled. The median age was 82 years (interquartile range 74.5-93.5). Ultrasound alterations were found in all patients enrolled; inhomogeneous intersti-
\end{abstract}

tial syndrome with spared areas (91.9\%) and pleural alterations $(100 \%)$ were the most frequent findings. At univariate analysis, LUS score (hazard ratio [HR] 1.168, 95\% Cl 1.0491.301) and pleural effusions (HR 3.995, 95\% Cl 1.056-15.110) were associated with in-hospital death. At multivariate analysis, only LUS score (HR 1.168, 95\% Cl 1.049-1.301) was independelty associated with in-hospital death. The LUS score's best cutoff for distinguishing patients experiencing in-hospital death was 17 (at multivariate analysis LUS score $\geq 17, \mathrm{HR}$ 4.827, 95\% Cl 1.452-16.040). In-hospital death was significantly different according to the LUS score cutoff of 17 ( $p=$ 0.0046). Conclusion: LUS could play a role in the diagnosis and prognosis in elderly patients hospitalized for SARSCoV-2 infection.

(c) 2020 S. Karger AG, Basel

\section{Introduction}

Within 3 months of the first case of novel coronavirus disease (COVID-19) >200,000 infected people and $>30,000$ deaths were documented in Italy. This infection represents a serious challenge to public health and the efficiency of the health care structures [1]. 
Higher rates of severe outcomes in patients with COVID-19 were found in patients aged 65 or more [2]. In particular, a higher mortality was detected in elderly patients due to a higher rate of comorbidities and frailty [3]. Recent evidence suggests that the earlier we treat, the better patients improve with treatment. Lung ultrasound (LUS) is a useful diagnostic tool in determining both lung involvement and its degree, thus potentially playing a role in treatment decisions [4]. Indeed, LUS is a user-friendly, noninvasive real-time tool available at the patient's bedside [5].

To date, the role of LUS has yet to be explored. Only few reports evaluated the clinical value of LUS during COVID-19 pandemic with promising results $[4,6,7]$.

Thickening of the pleural line with pleural line irregularity, B-lines in a variety of patterns including focal, multifocal, and confluent and consolidations were the most frequently found ultrasound features in patients with COVID-19 pneumonia.

However, to our knowledge, scarce and not definitive data are available regarding the role of LUS in elderly patients with COVID-19. Thus, we aimed to evaluate the diagnostic role of LUS in elderly patients admitted to the geriatric ward with severe acute respiratory syndromecoronavirus-2 (SARS-CoV-2) pneumonia. The secondary aim of the study was to correlate LUS findings with in-hospital death.

\section{Methods}

Patients aged $>65$ years were consecutively included in the study between April 6, 2020, and May 20, 2020 and were admitted to the geriatric ward of S. Orsola-Malpighi University Hospital, Bologna, Italy. Patients with respiratory symptoms on admission in the emergency department, a diagnosis of COVID-19 based on the detection of SARS-CoV-2 on RT-PCR from the nasopharyngeal swab and with available chest X-ray (CXR) or high-resolution computed tomography (HRCT) performed in the emergency department were included.

To minimize the possible interference on the LUS evaluation of other diseases, patients with the following conditions have been excluded: (a) chronic obstructive pulmonary disease history; (b) previous diagnosis of tuberculosis; (c) diagnosis of diffuse parenchymal lung disease (DPLD); and (d) diagnosis of lung neoplasia or pulmonary metastases from other neoplasia. In addition, another exclusion criteria were the impossibility to perform the ultrasound due to patient's opposition.

Data from the medical records of each patients were used; in particular demographics, laboratory exams, past medical history, and the clinical findings of the patients related to the hospitalization. Multimorbidity was assessed using the Charlson comorbidity index [8]. A disability evaluation was assessed by the number of preserved basic activities of daily living (ADL) [9] and by the

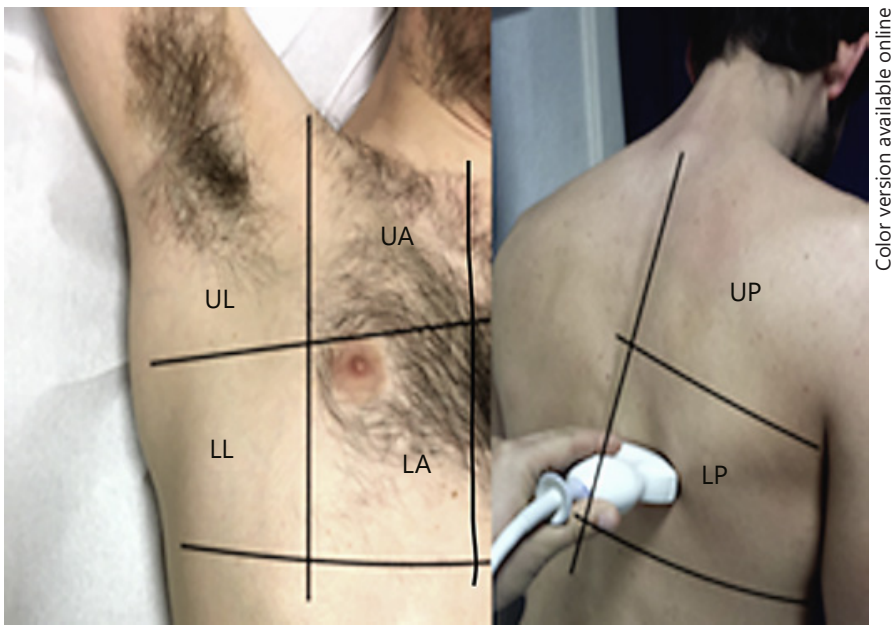

Fig. 1. Subdivision in chest areas. UA, upper anterior; LA, lower anterior; UL, upper lateral; LL, lower lateral; UP, upper posterior; $\mathrm{LP}$, lower posterior.

number of preserved instrumental activities of daily living (IADL) [10].

All patients enrolled were evaluated with LUS at admission to the geriatric ward. Patients' follow-up started at geriatric ward admission and was carried out until hospital discharge or death.

The study was conducted according to the Declaration of Helsinki's ethical principles for medical research involving human subjects. The protocol was reviewed and approved by the Ethics Committee of the S. Orsola-Malpighi Hospital in April 2020 (protocol number 1957/2020). Patient informed consent was obtained from each patient.

\section{Lung Ultrasound}

LUS was performed using a MyLab gamma echographic equipment (Esaote) by a physician (GR) of Bologna University Hospital with expertise in LUS with a standardized protocol [11].

Our protocol provides the use of both a linear ( 5 to $8 \mathrm{MHz}$ ) and a convex ( 2 to $5 \mathrm{MHz}$ ) probe. The depth and frequency are tailored to the patient. The focal point was set on the pleural line. With intercostal scan, the physician analyzed 12 chest areas for patient $(6$ for side: upper anterior, lower anterior, upper lateral, lower lateral, upper posterior, and lower posterior). The anterior and lateral area were separated by anterior axillary line, the lateral and posterior area were separated by posterior axillary lines (Fig. 1). The anterior scans were made following the midclavicular line, the lateral scans were made following the midaxillary line, and the posterior scans were made following the scapular line. The patient was in supine position to study the antero-lateral lung fields and in seated position for the analysis of the posterior lung areas. For patients who were not able to maintain the sitting position independently, this position was achieved with the help of a second operator (one operator performing LUS and the other helping the patient to maintain the seating position). According to the recommendations of the International Consensus Conference on LUS [11] for each area the physician analyses the following features: presence of interstitial syndrome (B-lines, not confluent or confluent vertical artifacts with white lung), distribution of B-lines (homogeneous or 
inhomogeneous with spared areas), consolidations and their distribution, pleural line abnormalities (irregular/fragmented, thickened, and/or with subpleural small echo poor lesions) and their distribution, pleural effusion, presence of atelectasis, and presence of lung sliding (normal, reduced or absent). Moreover, the physician recorded in the ultrasound report whether 2 operators were required for completing LUS as in the case of the patient with reduced motility and the time needed to perform the exam.

\section{LUS Score}

Lung ultrasound score (LUS score) [12], a useful tool in intensive care for monitoring patients with adult respiratory distress syndrome, was used in assessing severity of lung involvement. For each of the 12 chest areas a point scoring system is employed: ultrasound pattern -0 points (normal aeration line sliding sign associated with respiratory movement or $<3$ B-lines), 1 point (a clear number of visible B-lines with horizontal spacing between adjacent B-lines $\leq 7 \mathrm{~mm}$ ), 2 points (multiple B-lines fused that were difficult to count, including "white lung"), and 3 points (pulmonary consolidation). The final score of the patient was the sum of each regional ultrasound score (ranging from 0 to 36 ).

\section{Statistical Analysis}

Continuous variables were reported as median with interquartile range, while categorical variables, as numbers and percentages. Comparison of demographics, laboratory test, instrumental data (LUS and X-ray) among the 2 group of patients identified (patients with or without in-hospital death) were analyzed by Fischer, $\chi^{2}$, or Mann-Whitney tests. Subsequently, the same variables were tested as independent variables associated with in-hospital death. First, several univariate Cox regression analyses were performed considering all the variables. Subsequently, only the variables significantly associated with the in-hospital death in univariate analyses were entered into a multivariate model. Finally, the best multivariate model was identified, adopting a backward elimination procedure. The estimated hazard ratios (HR) with their $95 \%$ confidence intervals $(95 \% \mathrm{CI})$ were calculated; $p$ values $<0.05$ were considered statistically significant. The best cutoff for the LUS score for in-hospital death prediction according to the Liu method was calculated; the accuracy, sensitivity, and specificity were also reported. According to this cutoff value, the included patients were divided into 2 subgroups; then, using the Kaplan-Meier approach the risk of in-hospital death was estimated and compared between the 2 subgroups. The log-rank test was used for these comparisons. Statistical analyses were performed using Stata/SE (Version 13.0; Stata Corp, TX, USA) for Windows.

\section{Results}

A total of 37 patients (19 men) were enrolled in our study. The median age was 82 years (74.5-93.5). The median value of Charlson comorbidity index was 5 (1.5-7). About disability of our population the median of ADL was $1(0-6)$ and of IADL was 0 (0-8). Demographic characteristics, clinical features, and comorbidities are detailed in Table 1.
Table 1. Description of the population of our study. Demographic, clinical characteristics at admission, comorbidity, and disability

\begin{tabular}{|c|c|}
\hline & $\begin{array}{l}\text { Patients } n=37 n(\%) \text { or } \\
\text { median (IQR) }\end{array}$ \\
\hline Men, $n$ & $19(51.4)$ \\
\hline Age & $82(74.5-93.5)$ \\
\hline $\mathrm{BMI}, \mathrm{k} / \mathrm{m}^{2}$ & $23.7(22.5-26.4)$ \\
\hline Charlson comorbidity index & $5(1.5-7)$ \\
\hline $\mathrm{ADL}$ & $1(0-6)$ \\
\hline IADL & $0(0-8)$ \\
\hline Cognitive impairment & $16(43.2 \%)$ \\
\hline Hypertension & $23(62.2 \%)$ \\
\hline Coronary heart disease & $6(16.2 \%)$ \\
\hline Heart failure & $7(18.9 \%)$ \\
\hline CKD & $5(13.5 \%)$ \\
\hline Previous stroke & $4(10.8 \%)$ \\
\hline Diabetes & $6(16.2 \%)$ \\
\hline Respiratory rate & $20(17.5-24)$ \\
\hline $\mathrm{SpO}_{2} \%$ & $96(95-98.5)$ \\
\hline $\mathrm{PaO}_{2} / \mathrm{FiO}_{2}$ & $332.5(277-379)$ \\
\hline Fever & $19(51.4)$ \\
\hline Dyspnea & $14(37.8)$ \\
\hline Cough & $9(24.3)$ \\
\hline Systolic blood pressure & $115(106-130)$ \\
\hline Hospitalization duration, days & $7(5-10.5)$ \\
\hline In-hospital death & $11(29.7 \%)$ \\
\hline
\end{tabular}

IQR, interquartile range; ADL, activities of daily living; IADL, instrumental activities of daily living.

\section{LUS}

Abnormal LUS findings were recorded in all patients enrolled. The average time required for the examination was $9.6 \mathrm{~min}$ (2.5). In $48.6 \%$ of cases, 2 operators were needed to complete the LUS.

All patients had an interstitial syndrome (B-lines); 36 (97.3\%) with bilateral involvement and 34 patients (91.9\%) with inhomogeneous distribution with spared areas. White lung areas were found in 12 subjects (32.4\%). Pleural line abnormalities were detected in all patients. In $70.3 \%$ these alterations were found with diffuse distribution. Thirty-three patients (89.2\%) presented a fragmented pleural line, 15 (40.5\%) thickened pleural line, and 22 (59.5\%) had subpleural small echo poor lesions. Coexistence of fragmented and thickened pleural line was found in $13(35.1 \%)$ patients, while the association between fragmented pleural line and subpleural small echo poor lesions were found in 18 (48.6\%) cases. Coexistence of thickened pleural line and subpleural consolidation was detected in $9(24.3 \%)$ subjects. Finally, coexistence of fragmented, thickened pleural line and subpleural small
Recinella et al. 
Table 2. LUS findings in the 37 patients enrolled

\begin{tabular}{lc}
\hline LUS features & $\begin{array}{c}\text { Results, } n(\%) \text { or } \\
\text { median (IQR) }\end{array}$ \\
\hline Abnormal ultrasound finding & $37(100 \%)$ \\
Median time for exam, min & $10(8-12)$ \\
Two operators needed & $18(48.6 \%)$ \\
Interstitial syndrome (B-lines) & $37(100 \%)$ \\
Bilateral interstitial syndrome & $36(97.3 \%)$ \\
With spared areas & $34(91.9 \%)$ \\
White lung area & $12(32.4 \%)$ \\
Pleural line abnormalities & $37(100 \%)$ \\
(a) Fragmented pleural line & $33(89.2 \%)$ \\
(b) Thickened pleural line & $15(40.5 \%)$ \\
(c) Subpleural small echo poor lesions & $22(59.5 \%)$ \\
a + b & $13(35.1 \%)$ \\
a + c & $18(48.6 \%)$ \\
b + c & $9(24.3 \%)$ \\
a + b c c & $7(18.9 \%)$ \\
B-Lines + pleural line abnormalities & $100 \%$ \\
Consolidations & $17(45.9 \%)$ \\
Bilateral distribution & $41.2 \%$ \\
Pleural effusion & $10(27 \%)$ \\
Bilateral localization & $70 \%$ \\
Normal lung sliding & $30(81.1 \%)$ \\
Reduced lung sliding & $7(18.9 \%)$ \\
Abolish ling sliding & 0 \\
LUS score & $12(8-17)$ \\
\hline
\end{tabular}

IQR, interquartile range; LUS, lung ultrasound.

echo poor lesions was found in 7 (18.9\%) patients. In all patients enrolled we found an association between interstitial syndrome and pleuric alteration. We found consolidations in 17 (45.9\%) subjects, of whom $41.2 \%$ were bilateral.

Pleural effusion was found in $10(27 \%)$ cases (bilateral in $70 \%$ of them) while atelectasis only in $2(5.4 \%)$ of patients. Lung sliding appeared to be reduced in 7 (18.9\%). LUS score was estimated in all patients and the median value was 12 (8-17). LUS features were summarized in Table 2.

\section{Imaging Evaluations}

Of the 37 patients enrolled, 25 underwent CXR and the other 12 underwent HRCT (Table 3). About CXR findings, among 25 patients with CXR, 6 (24\%) had a normal examination. Interstitial pattern was found in $12(48 \%)$ of CXR (bilateral in $66.7 \%$ of cases). Consolidations were found in 7 (28\%) of CXR (42.9\% of these were bilateral). Pleural effusion was found only in 4 cases ( $16 \%$ of CXR) and in one of these cases bilateral. Abnormal radiological
Table 3. Results of CXR and HRCT examinations

\begin{tabular}{lc}
\hline CXR findings & $\begin{array}{l}\text { Results, } n(\%) \text { or } \\
\text { median (IQR) }\end{array}$ \\
\hline CXR findings & \\
Number of patients that underwent CXR & 25 \\
Patient with abnormal CXR findings & $19(76 \%)$ \\
Interstitial pattern & $12(48 \%)$ \\
$\quad$ Bilateral involvement & $66.7 \%$ \\
Consolidations & $7(28 \%)$ \\
$\quad$ Bilateral consolidations & $42.9 \%$ \\
Pleural effusion & $34(16 \%)$ \\
$\quad$ Bilateral pleural effusion & $25 \%$ \\
\hline HRCT findings & \\
Number of patients that underwent HRCT & 12 \\
Patients with abnormal HRCT findings & $12(100 \%)$ \\
Interstitial pattern & $10(83.3 \%)$ \\
$\quad$ Ground-glass pattern & $100 \%$ \\
Bilateral involvment & $90 \%$ \\
Consolidations & $3(25 \%)$ \\
$\quad$ Bilateral consolidations & $0(0 \%)$ \\
Pleural effusion & $1(8.3 \%)$ \\
$\quad$ Bilateral pleural effusion & $100 \%$ \\
\hline
\end{tabular}

IQR, interquartile range; CXR, chest X-Ray; HRCT, high-resolution computed tomography.

findings were found in all 12 patients who underwent HRCT. Interstitial pattern was found in $10(83.3 \%)$ patients (all of them with ground-glass opacities). In 3 (25\%) patients consolidations were also reported.

\section{In-Hospital Death}

During the follow-up, 11 (29.7\%) patients died after a median time of hospitalization of 8 days (5-13). The remaining 26 patients were discharged after a median hospitalization of 7 (5-10.2) days.

Differences in demographic, clinical, laboratory tests, and LUS characteristics among the group of patients undergoing or not undergoing in-hospital death were reported in Table 4. In-hospital death was associated with lower BMI $(p=0.039)$ and with lower values of GFR ( $p=$ $0.009)$. Regarding LUS features, patients undergoing inhospital death showed a higher prevalence of reduced lung sliding $(p=0.007)$ and higher values of LUS score $(p=0.028)$. Charlson comorbidity index, ADL, and IADL were similar across the 2 groups $(p=0.305, p=0.624$, and $p=0.379$, respectively).

Among all variables evaluated in the univariate Cox regression analysis (Table 5), LUS score (HR 1.168 [CI 1.049-1.301]) and pleural effusions (HR 3.995 [CI 1.056- 
Table 4. Differences in demographic, clinical, and LUS characteristics undergoing or not undergoing in-hospital death

\begin{tabular}{|c|c|c|c|}
\hline & $\begin{array}{l}\text { Survivors }(n=26) \\
n(\%) \text { or } \\
\text { median }(\mathrm{IQR})\end{array}$ & $\begin{array}{l}\text { In-hospital death } \\
(n=11), n(\%) \text { or } \\
\text { median (IQR) }\end{array}$ & $p$ value \\
\hline Men & $15(57.7)$ & $4(36.4)$ & 0.235 \\
\hline Age & $80.5(73.7-92.2)$ & $90(82-95)$ & 0.083 \\
\hline $\mathrm{BMI}, \mathrm{kg} / \mathrm{m}^{2}$ & $24.9(22.7-28.3)$ & $22.9(21.7-23.7)$ & 0.036 \\
\hline Respiratory rate & $20(18-24)$ & $20(16-24)$ & 0.913 \\
\hline $\mathrm{SpO}_{2} \%$ & $96.5(95-99)$ & $94(93-97)$ & 0.658 \\
\hline Dyspnea & $11(29.7)$ & $3(8.1)$ & 0.477 \\
\hline Cough & $6(16.2)$ & $3(8.1)$ & 0.857 \\
\hline Fever & $15(40.5)$ & $4(10.8)$ & 0.295 \\
\hline Charlson comorbidity index & $4(0.7-7)$ & $5(4-7)$ & 0.305 \\
\hline ACE inhibitors & $7(26.9)$ & $2(18.2)$ & 0.682 \\
\hline Sartans & $4(15.4)$ & $0(0)$ & 0.481 \\
\hline $\mathrm{ADL}$ & $3(0-6)$ & $1(0-6)$ & 0.624 \\
\hline IADL & $1.5(0-8)$ & $0(0-8)$ & 0.379 \\
\hline Cognitive impairment & $12(46.2)$ & $4(36.4)$ & 0.583 \\
\hline $\mathrm{PaO}_{2} / \mathrm{FiO}_{2}$ at entrance & $336(265-378)$ & $329(282-380)$ & 0.757 \\
\hline White blood cells, $\times 10^{9} / \mathrm{L}$ & $6.76(5.04-10.09)$ & $8.79(5.87-12.45)$ & 0.238 \\
\hline Lymphocytes, $\times 10^{9} / \mathrm{L}$ & $1.30(0.90-1.51)$ & $1.38(0.28-1.67)$ & 0.868 \\
\hline Thrombocytes, $\times 10^{9} / \mathrm{L}$ & $196(159-243)$ & $219(141-227)$ & 0.961 \\
\hline C-reactive protein, $\mathrm{mg} / \mathrm{dL}$ & $8.16(2.93-13.46)$ & $4.18(2.58-15.54)$ & 0.455 \\
\hline D-dimer, $\mu \mathrm{g} / \mathrm{mL}$ & $1.05(0.30-2.91)$ & $0.86(0.32-2.84)$ & 0.770 \\
\hline Ferritin, ng/mL & $191(88.5-438)$ & $195(27-1,032)$ & 0.786 \\
\hline $\mathrm{LDH}, \mathrm{U} / \mathrm{L}$ & $217(135-440)$ & $217(162-254)$ & 0.796 \\
\hline $\mathrm{GRF}, \mathrm{mL} / \mathrm{min}$ & $68(36.2-92.2)$ & $12(8-53)$ & 0.009 \\
\hline Albumin, g/L & $31.3(26-34.7)$ & $24.4(13.7-30.6)$ & 0.126 \\
\hline \multicolumn{4}{|l|}{ LUS characteristics } \\
\hline White lung areas & $6(23.1)$ & $6(54.5)$ & 0.062 \\
\hline Fragmented pleural line & $22(84.6)$ & $11(100)$ & 0.168 \\
\hline Thickened pleural line & $9(34.6)$ & $6(54.5)$ & 0.259 \\
\hline Subpleural small echo poor lesions & $14(53.8)$ & $8(72.7)$ & 0.285 \\
\hline Consolidations & $11(42.3)$ & $6(54.5)$ & 0.495 \\
\hline Pleural effusion & $5(19.2)$ & $5(45.5)$ & 0.101 \\
\hline Reduced lung sliding & $2(7.7)$ & $5(45.5)$ & 0.007 \\
\hline LUS score & $11(7-14)$ & $18(11-21)$ & 0.028 \\
\hline
\end{tabular}

IQR, interquartile range; ACE, angiotensin converting enzyme; LDH, lactate dehydrogenases; GRF, glomerular filtration rate; LUS, lung ultrasound.

15.110]) were associated with in-hospital death. Multivariate Cox analysis showed that only LUS score was independently associated with in-hospital death (HR 1.168 [CI 1.049-1.301], $p=0.004$ ).

LUS score's best cutoff for the prediction of in-hospital death was 17 (specificity 88\%, sensitivity 55\%, and accuracy $72 \%$ ). At univariate Cox regression analysis, the dichotomized LUS score $(\geq 17)$ was associated with in-hospital death and resulted in the only predictor of the event even at multivariate analysis (HR 4.827 [CI 1.452-16.040], $p=0.010)$. Kaplan-Meier survival curves according to the
LUS score cutoff value were estimated (Fig. 2). In-hospital death is shown in the subgroup of patients with LUS score $\geq 17$ compared to those with LUS score $<17$, with a statistically significant difference $(p=0.004)$.

\section{Discussion}

The COVID-19 emergency has put a strain on Italian hospitals. A massive reorganization of the wards was necessary to deal with this emergency [13]. Moreover, simi- 
Table 5. Univariate and multivariate analyses for independent variables associated with in-hospital death

\begin{tabular}{|c|c|c|c|c|}
\hline \multirow[t]{2}{*}{ In-hospital death $(n=11)$} & \multicolumn{2}{|l|}{ Univariate } & \multicolumn{2}{|l|}{ Multivariate } \\
\hline & $\operatorname{HR}(95 \% \mathrm{CI})$ & $p$ value & HR (95\% CI) & $p$ value \\
\hline Sex & $0.556(0.160-1.927)$ & 0.355 & & \\
\hline Age & $1.039(0.969-1.115)$ & 0.274 & & \\
\hline Cognitive impairment & $0.542(0.157-1.875)$ & 0.334 & & \\
\hline $\mathrm{ADL}$ & $0.974(0.776-1.223)$ & 0.826 & & \\
\hline IADL & $0.974(0.823-1.153)$ & 0.762 & & \\
\hline Charlson comorbidity index & $1.065(0.879-1.291)$ & 0.517 & & \\
\hline $\mathrm{BMI}$ & $0.905(0.770-1.064)$ & 0.231 & & \\
\hline White blood cells & $1.127(0.978-1.299)$ & 0.097 & & \\
\hline Lymphocytes & $1.236(0.421-3.625)$ & 0.699 & & \\
\hline GFR & $0.979(0.958-1.000)$ & 0.052 & & \\
\hline LDH & $0.997(0.994-1.001)$ & 0.264 & & \\
\hline Ferritin & $0.999(0.997-1.000)$ & 0.449 & & \\
\hline C-reactive protein & $0.946(0.856-1.045)$ & 0.275 & & \\
\hline Albumin & $0.846(0.705-1.015)$ & 0.073 & & \\
\hline $\mathrm{PaO}_{2} / \mathrm{FiO}_{2}$ & $1.001(0.996-1.005)$ & 0.680 & & \\
\hline \multicolumn{5}{|l|}{ LUS } \\
\hline LUS score & $1.168(1.049-1.301)$ & 0.004 & $1.168(1.049-1.301)$ & 0.004 \\
\hline White lung areas & $2.534(0.770-8.339)$ & 0.126 & & \\
\hline Consolidations & $1.047(0.316-3.465)$ & 0.939 & & \\
\hline Pleural effusions & $3.995(1.056-15.110)$ & 0.041 & & \\
\hline Reduced lung sliding & $1.672(0.456-6.134)$ & 0.438 & & \\
\hline
\end{tabular}

ADL, activities of daily living; IADL, instrumental activities of daily living; GFR, glomerular filtration rate; LDH, lactate dehydrogenases; LUS, lung ultrasound.

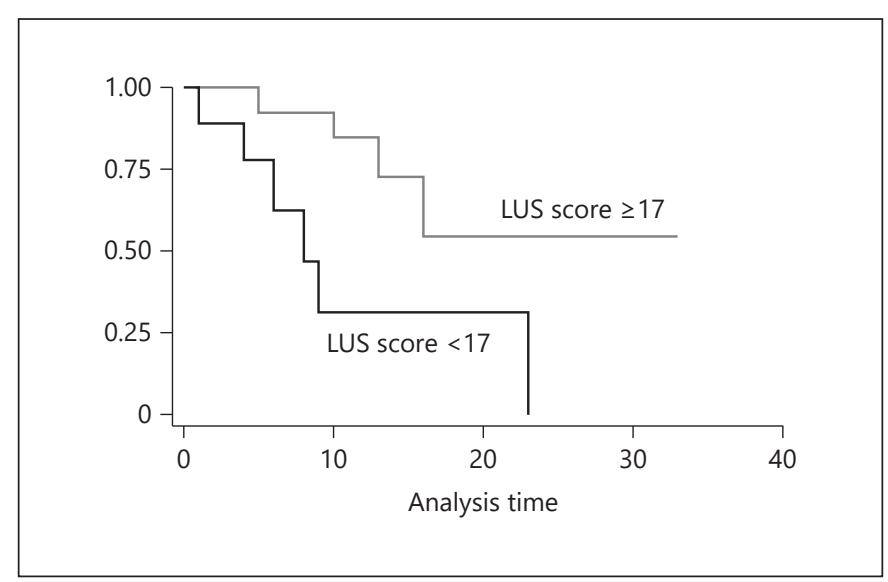

Fig. 2. Kaplan-Meier survival curves according to the LUS score cutoff. LUS, lung ultrasound.

larly to other countries, in Italy, the population most affected in terms of in-hospital mortality were the elderly due to the presence of multiple comorbidities and frailty [14]. In addition, the rapid diffusion and progression of
COVID-19 was translated in the clinical setting in the need of a tool able to guarantee an early identification and to allow a prospective monitoring of the disease; this ideal tool would provide an accurate and rapidly imaging evaluation able to stratify patient's prognosis and disease severity, taking also into account the logistic issues related to the infective risks.

To our knowledge, this is the first study evaluating ultrasound characteristics in an elderly population with SARS-CoV-2 pneumonia and assessing its prognostic value. For this purpose, we enrolled an elderly population with a high degree of comorbidity and disability as demonstrated by the median values of Charlson comorbidity index, ADL, and IADL.

Previous studies in non-age-selected populations reported that COVID-19 pneumonia lesions had a typically peripheral distribution [15] that makes ultrasound detection relatively easy. The ultrasound features most commonly found in patients with SARS-CoV-2 pneumonia were the presence of interstitial syndrome (B-lines) with generally inhomogeneous distribution (mixing A and $B$ patterns nearby) [16] and irregular pleural line with 


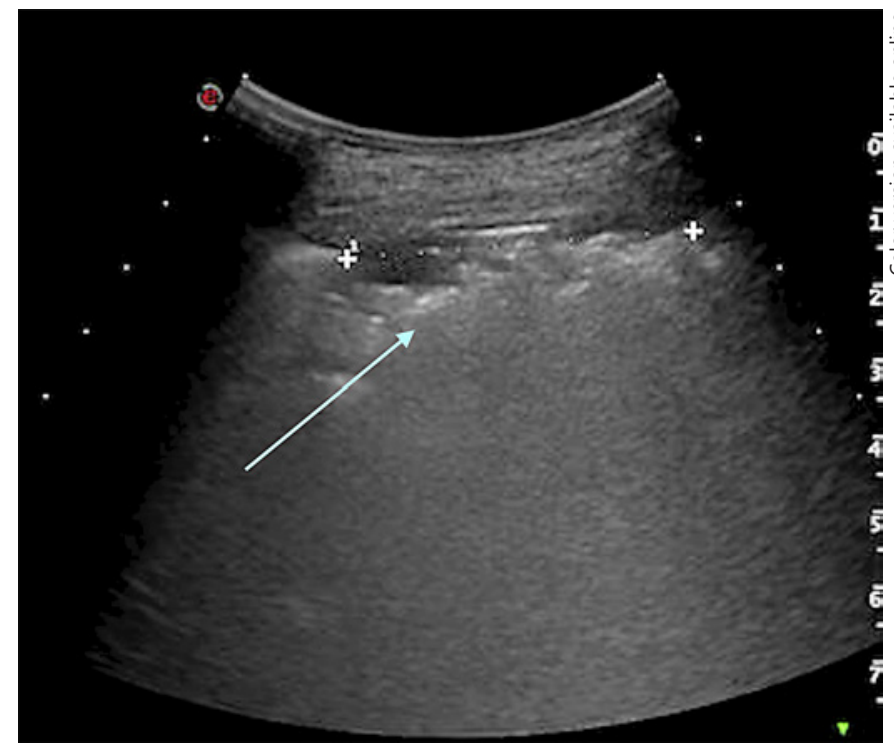

Fig. 3. LUS image of fragmented pleural line with subpleural small echo poor lesions. LUS, lung ultrasound.

the presence of thickening and subpleural small consolidations (Fig. 3). With increasing severity, areas of white lung can be observed (Fig. 4) up to the presence of big consolidations with air bronchogram [6] (Fig. 5).

We asked ourselves whether diagnostic and prognostic accuracy could be limited in the elderly with COVID-19. In fact the senile lung has been found to be a possible source of pitfalls for LUS. Furthermore, elderly patients may have reduced compliance during the examination. For example, they could be unable to understand orders because of cognitive impairment.

Our findings confirmed the promising role of LUS in the diagnosis and management of patients with $\mathrm{CO}$ VID-19 [17], even in the elderly. In fact, we found suggestive ultrasound signs of SARS-CoV-2 interstitial pneumonia in all enrolled patients. In particular, the high prevalence of interstitial syndrome (B-lines) with inhomogeneous distribution and pleural line alterations was confirmed.

There was also a high prevalence of consolidations and white lung areas. However, these 2 ultrasound signs did not show any statistical significance in predicting in-hospital death.

Probably due to the immune senescence, we found a lower prevalence of respiratory symptoms than that in the adult population. This could further emphasize the role of LUS, which, even in patients without respiratory symptoms, is able to find suggestive alterations for interstitial pneumonia.

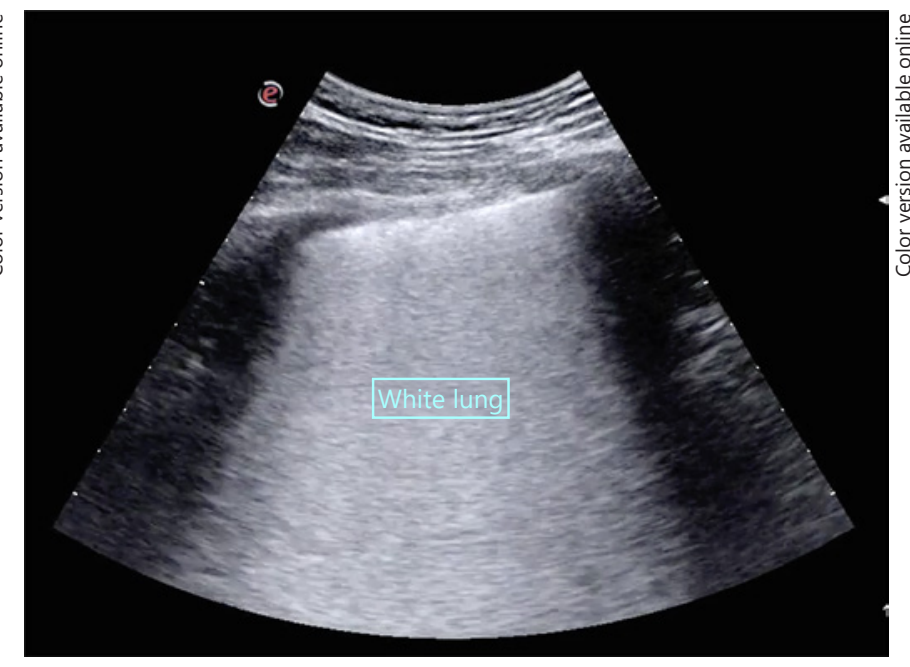

Fig. 4. LUS image of "white lung." LUS, lung ultrasound.

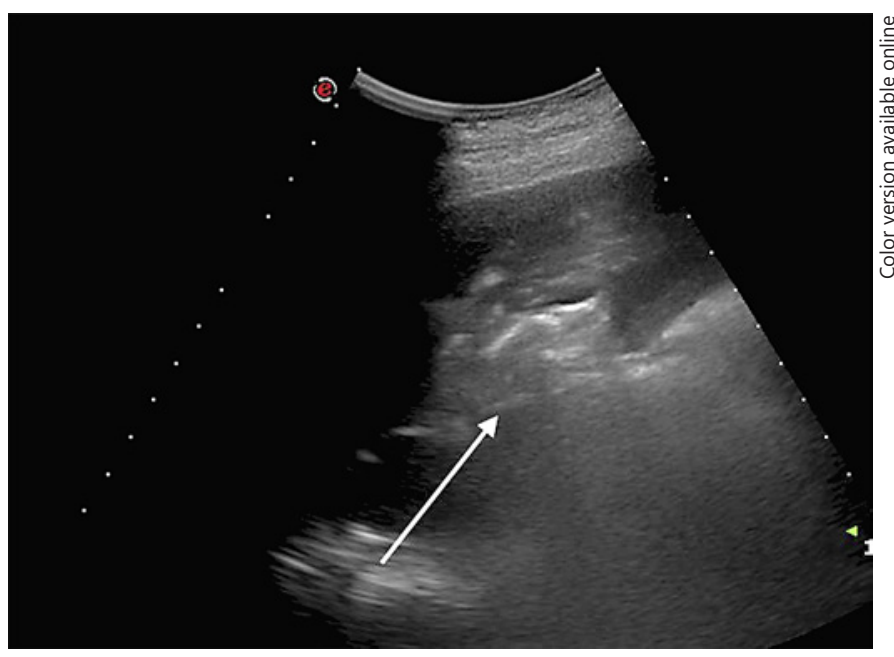

Fig. 5. LUS image of consolidations with air bronchogram. LUS, lung ultrasound.

The prognostic role of LUS was also assessed. For this purpose, we used an ultrasound score previously validated in literature: the LUS score [12]. We found higher values of LUS score in the group of patients undergoing inhospital death.

Moreover, the in-hospital death rate was significantly higher in patients with kidney failure; in 5 cases there was a worsening of chronic renal failure, while in the remaining 6 cases there was no known history of renal failure. Thus, there may be a correlation between ultrasound alterations and renal failure since fluid congestion secondary to renal failure could result in an increased number of $\mathrm{B}$-lines and therefore a higher LUS-score value.
Recinella et al. 
The strong predictive value of LUS score for assessing survival was confirmed at the univariate and multivariate analysis (HR 1.168 [CI 1.049-1.301)] since it resulted in the only predictor of survival. Furthermore, we were able to provide a LUS score cutoff of 17 for identifying those patients at higher risk of in-hospital death.

Thus, according to our data, we strongly advice the use of LUS at COVID-19 patient admission for evaluating patient's disease degree and death risk in order to carry out a therapeutic and overall management of patient-tailored approach based on LUS. Among the advantages of LUS there is the possibility to perform it at bedsides, which according to our opinion minimizes the infective risks linked to patient's transfer to radiology units for performing CXR or HRCT, limiting the number of healthcare workers exposed to contamination and saving personal protective equipment [18]. On the other hand, the singleoperator LUS examiner approaches the patient with all personal protective equipment including face masks, gloves, disposable caps, shoe covers, protective glasses or goggles, or face barriers. Moreover, LUS is a repeatable technique that can be used even during patients' followup since it has no costs and to avoid patients' radiation exposure.

Indeed, as regard other imaging modalities for diagnosis and follow-up of COVID-19 patients, CXR is a commonly used imaging technique in suspected COVID-19. However, the image quality is often poor, especially in elderly patients who are often uncooperative [19]. In accordance with this observation, differently from what was said for the ultrasound, where alterations were found in all patients, the radiography showed pathological signs only in $76 \%$ of patients. In our study, we have enrolled only a few patients undergoing HRCT so drawing conclusions seems to be hazardous. However, all HRCT patients had pathological imaging patterns and in particular ground-glass opacities were the most frequently found sign.

Our study has some limitations: first, the small sample size of patients enrolled was due to several reasons: elderly patients were also admitted in internal medicine units, thus different from the geriatric one; second, the study began after the COVID-19 peak in Emilia-Romagna, thus preventing to enroll a large number of patients for a limited number of daily hospitalization; third, patients were often sent to geriatric ward without CXR or HRCT and thus not suitable for the study, sometimes in critical conditions which did not allow to move the patient in the radiology unit for performing imaging. Moreover, we did not evaluate the intra- and interoperator variability for minimizing the infective risks and to save personal protective equipment. Finally, our multivariate analysis may be affected by an overfitting variable bias since only 11 patients underwent in-hospital death.

Among the limitations of LUS evaluation, even if not evaluated in the present study, the low specificity for identifying a single disease should be mentioned. In the setting of our study, the main LUS findings of COVID-19 patients have also been previously reported in a range of other alveolar-interstitial syndromes.

Our study has several strengths: we reported from a sample of consecutively enrolled elderly patients that ultrasound plays a key role in recognizing the degree of lung involvement in SARS-CoV-2 pneumonia; moreover, to our knowledge, this is the first study evaluating the prognostic value of LUS in elderly COVID-19 patients, finding that this technique is able to stratify the risk in elderly patients hospitalized for interstitial pneumonia by SARS-CoV-2. Future studies including a larger sample size and comparing ultrasound characteristics and HRCT features in elderly subjects are needed. Moreover, validation studies of the identified cutoff of the LUS score would be desirable.

\section{Conclusion}

LUS is playing a key role in the in-hospital emergency from COVID-19. Our study highlights the diagnostic role of LUS in SARS-CoV-2 pneumonia also in elderly patients with a high degree of disability and comorbidity. LUS score showed a prognostic role in stratifying the risk of in-hospital death.

\section{Statement of Ethics}

The study approved by the Ethics Committee of the S. OrsolaMalpighi Hospital in April 2020 (protocol number 1957/2020), Bologna, Italy according to the ethical guidelines of the 1975 Declaration of Helsinki. Subjects (or their parents or guardians) have given their written informed consent.

\section{Conflict of Interest Statement}

The authors declare no conflicts of interest.

\section{Funding Sources}

The authors did not receive any funding. 


\section{Author Contributions}

Guerino Recinella was responsible for study design. Guerino Recinella, Giovanni Marasco, and Manuel Tufoni were responsible for data collection and analysis. Mara Brizi, Eleonora Evange- listi, Lorenzo Maestri, Marco Fusconi, Pietro Calogero, and Donatella Magalotti contributed to the data collection and revision of the manuscript. Marco Zoli contributed substantially to the study design and review of the manuscript.

\section{References}

1 Smith MJ, Hayward SA, Innes SM, Miller A. Point-of-care lung ultrasound in patients with COVID-19: a narrative review. Anaesthesia. 2020 Aug;75(8):1096-104.

2 Grasselli G, Zangrillo A, Zanella A, Antonelli $\mathrm{M}$, Cabrini L, Castelli A, et al. Baseline characteristics and outcomes of 1591 patients infected with SARS-CoV-2 admitted to ICUs of the Lombardy region, Italy. JAMA. 2020 Apr; 323(16):1574-81.

3 Wang D, Yin Y, Hu C, Liu X, Zhang X, Zhou S, et al. Clinical course and outcome of 107 patients infected with the novel coronavirus, SARS-CoV-2, discharged from two hospitals in Wuhan, China. Crit Care. 2020 Apr;24(1): 188.

4 Soldati G, Smargiassi A, Inchingolo R, Buonsenso D, Perrone T, Briganti DF, et al. Proposal for international standardization of the use of lung ultrasound for COVID-19 patients; a simple, quantitative, reproducible method. J Ultrasound Med. 2020 Jul;39(7): 1413-9.

5 Vizioli L, Forti P, Bartoli E, Giovagnoli M, Recinella G, Bernucci D, et al. Accuracy of lung ultrasound in patients with acute dyspnea: the influence of age, multimorbidity and cognitive and motor impairment. Ultrasound Med Biol. 2017 Sep;43(9):1846-52.

6 Peng QY, Wang XT, Zhang LN. Findings of lung ultrasonography of novel corona virus pneumonia during the 2019-2020 epidemic. Intensive Care Med. 2020 May;46(5):849-50.
7 Buonsenso D, Piano A, Raffaelli F, Bonadia N, de Gaetano Donati K, Franceschi F. Point-ofcare lung ultrasound findings in novel coronavirus disease-19 pnemoniae: a case report and potential applications during COVID-19 outbreak. Eur Rev Med Pharmacol Sci. 2020 Mar;24(5):2776-80.

8 Charlson ME, Pompei P, Ales KL, MacKenzie CR. A new method of classifying prognostic comorbidity in longitudinal studies: development and validation. J Chronic Dis. 1987; 40(5):373-83.

9 Katz S, Downs TD, Cash HR, Grotz RC. Progress in development of the index of ADL. Gerontologist. 1970;10(1):20-30.

10 Lawton MP, Brody EM. Assessment of older people: self-maintaining and instrumental activities of daily living. Gerontologist. 1969; 9(3):179-86.

11 Volpicelli G, Elbarbary M, Blaivas M, Lichtenstein DA, Mathis G, Kirkpatrick AW, et al. International evidence-based recommendations for point-of-care lung ultrasound. Intensive Care Med. 2012 Apr;38(4):577-91.

12 Zhao Z, Jiang L, Xi X, Jiang Q, Zhu B, Wang $M$, et al. Prognostic value of extravascular lung water assessed with lung ultrasound score by chest sonography in patients with acute respiratory distress syndrome. BMC Pulm Med. 2015 Aug;15:98.
13 Cecconi M, Piovani D, Brunetta E, Aghemo A, Greco M, Ciccarelli M, et al. Early Predictors of clinical deterioration in a cohort of 239 patients hospitalized for Covid-19 infection in Lombardy, Italy. J Clin Med. 2020 May; 9(5):E1548.

14 Bruno G, Perelli S, Fabrizio C, Buccoliero GB. Short-term outcomes in individuals aged 75 or older with severe coronavirus disease (COVID-19): first observations from an infectious diseases unit in Southern Italy. J Infect. 2020 Aug;81(2):e86-8.

15 Shi H, Han X, Jiang N, Cao Y, Alwalid O, Gu $\mathrm{J}$, et al. Radiological findings from 81 patients with COVID-19 pneumonia in Wuhan, China: a descriptive study. Lancet Infect Dis. 2020 Apr;20(4):425-34.

16 Volpicelli G, Gargani L. Sonographic signs and patterns of COVID-19 pneumonia. Ultrasound J. $2020 \mathrm{Apr} ; 12(1): 22$.

17 Soldati G, Smargiassi A, Inchingolo R, Buonsenso D, Perrone T, Briganti DF, et al. Is there a role for lung ultrasound during the COVID-19 pandemic? J Ultrasound Med. 2020 Jul;39(7):1459-62.

18 Mongodi S, Orlando A, Arisi E, Tavazzi G, Santangelo E, Caneva L, et al. Lung ultrasound in patients with acute respiratory failure reduces conventional imaging and health care provider exposure to COVID-19. Ultrasound Med Biol. 2020 Aug;46(8):2090-3.

19 Schultz MJ, Sivakorn C, Dondorp AM. Challenges and opportunities for lung ultrasound in novel coronavirus disease (COVID-19). Am J Trop Med Hyg. 2020 Jun;102(6):1162-3. 